\title{
س
}

$>\mathrm{DE}$

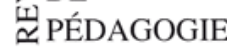

\author{
Recherches en éducation
}

$204 \mid 2018$

Varia

\section{BALUTEAU François, DUPRIEZ Vincent \& VERHOEVEN Marie (dir.). Entre tronc commun et filières, quelle école moyenne? Étude comparative}

Paris : Academia/L'Harmattan, 2018, 294 p.

Pierre Champollion

\section{OpenEdition}

Journals

Édition électronique

URL : https://journals.openedition.org/rfp/8492

DOI : $10.4000 /$ rfp.8492

ISSN : 2105-2913

Éditeur

ENS Éditions

Édition imprimée

Date de publication : 30 septembre 2018

Pagination : 82-84

ISBN : 979-10-362-0208-7

ISSN : 0556-7807

\section{Référence électronique}

Pierre Champollion, «BALUTEAU François, DUPRIEZ Vincent \& VERHOEVEN Marie (dir.). Entre tronc commun et filières, quelle école moyenne ? Étude comparative », Revue française de pédagogie [En ligne], 204 | 2018, mis en ligne le 30 septembre 2018, consulté le 13 janvier 2023. URL : http://

journals.openedition.org/rfp/8492 ; DOI : https://doi.org/10.4000/rfp.8492

Ce document a été généré automatiquement le 13 janvier 2023.

Tous droits réservés 


\section{BALUTEAU François, DUPRIEZ Vincent \& VERHOEVEN Marie (dir.). Entre tronc commun et filières, quelle école moyenne? Étude comparative}

Paris : Academia/L'Harmattan, 2018, 294 p.

Pierre Champollion

\section{RÉFÉRENCE}

BALUTEAU François, DUPRIEZ Vincent \& VERHOEVEN Marie (dir.). Entre tronc commun et filières, quelle école moyenne ? Étude comparative. Paris : Academia/L'Harmattan, 2018, $294 \mathrm{p}$.

Disons-le d'emblée, l'organisation interne de cet ouvrage nous paraît tout à fait pertinente sur le fond. Les présentations des différents systèmes éducatifs nationaux sont toutes regroupées deux par deux, en fonction de la progression visée de la thématique comparative. Les deux pays " germaniques », l'Allemagne et la Suisse (si on peut le dire pour le canton de Vaud!), introduisent dans une première partie le débat par la question centrale, on le verra, du « recours précoce aux filières ». Dans une deuxième partie, qui comporte l'Angleterre et le Québec, c'est la capacité d'« adaptation à la carte » des systèmes éducatifs qui est mise en avant. La troisième partie, au travers des systèmes éducatifs français et catalan, témoigne des inévitables "tensions autour de l'école commune» qu'alimentent contradictoirement différenciation curriculaire et «compréhensivité » scolaire. Une quatrième partie, centrée sur les cas de la Suède et de la Belgique, atteste, elle, les mouvements parfois opposés qui traversent les systèmes éducatifs : " construction d'une école commune » et « développement de filières éducatives ». 
2 Au-delà de ces quatre regroupements qui permettent au lecteur de cheminer dans la problématique générale de l'ouvrage qu'annonce justement le titre - «Entre tronc commun et filières, quelle école moyenne ?»-, introduction et conclusion ouvrent et nourrissent respectivement l'approche comparative. Mais, de par cette organisation interne originale, la volonté comparative affichée ne gomme pas - ce qui une bonne chose!- la présentation détaillée des différents systèmes éducatifs secondaires «moyens» effectuée au sein des différentes parties. Nous emprunterons le même cheminement pour présenter l'ouvrage, avant de nous attarder un peu plus longuement sur les éléments d'analyse comparative qu'il inclut et sur les apports au champ de la recherche en éducation qu'il contient.

3 Sur le plan de la forme, enfin, nous aurions apprécié que cet ouvrage bien écrit, aux intertitres explicites, apporte dans de brefs encadrés, au début de chaque partie ou mieux encore, en fin d'icelles, un bref résumé des éléments essentiels caractérisant chacune des huit écoles moyennes présentées, malgré la difficulté évidente de l'entreprise dans certains cas (notamment dans le cas des systèmes éducatifs non " centralisés "). Même si les conclusions internes à chaque " monographie " jouent un peu ce rôle, une telle initiative systématique eût sans doute facilité l'approche comparative visée...

4 À travers les monographies initiales de l'Allemagne et de la Suisse (canton de Vaud), c'est bien le «recours précoce aux filières " qui est d'abord mis en évidence. Cette opposition relative - relative parce que la «sélection latine » mise en œuvre par la pratique "sociale» du redoublement en France par exemple s'apparente par ses conséquences aux filières - se manifeste grosso modo entre la Mitteleuropa « germanique », d'un côté, et la Méditerranée et la Scandinavie - curieux attelage ! - de l'autre. Au-delà des deux exemples analysés, le recours précoce aux filières concerne aussi d'autres pays bien sûr, à commencer par l'Autriche voisine. Dans la deuxième partie, consacrée elle à l'Angleterre et au Québec, intitulée "Deux systèmes d'adaptation à la carte ", les auteurs illustrent le cheminement politique conduisant progressivement d'une logique participative à une logique marchande... En France et en Catalogne, ce sont surtout les "tensions autour de l'école commune», entre l'intégration de tous les élèves dans une même école - «compréhensivité " parfois essentiellement formelle - et la diversification des parcours, qui sont mises en avant. En Suède et en Belgique francophone, enfin, se font jour «des mouvements à contresens » (ou plutôt en sens inverse !). Les auteurs insistent entre autres à ce propos sur la prégnance de l'immigration dans le "revirement" suédois qui consacre le passage de la social-démocratie protectrice au néo-libéralisme et, sur le plan éducatif, de la pédagogie centrée sur l'élève à la gestion du curriculum par l'élève, ce qui ne manque pas d'accroitre le poids de l'origine sociale.

5 L'ouvrage comporte également plusieurs éléments d'analyse comparative qui, jalonnant les huit monographies, tentent de dégager régularités et oppositions entre elles. L'introduction, qui problématise la question, témoigne en premier lieu - au-delà des ordres scolaires cloisonnés hérités du XIX siècle - d’un mouvement général, mais pas systématique, menant au $\mathrm{xx}^{\mathrm{e}}$ siècle vers une plus grande intégration sociale par la scolarisation commune dans les mêmes établissements de toutes les couches de la société en Europe. Aujourd'hui, ce mouvement est - au moins partiellement - remis en question par la tendance généralisée dans presque tous les pays analysés - mais pas dans tous - à déconstruire l'école moyenne compréhensive difficilement bâtie. Dans 
leurs conclusions, les coordonnateurs de l'ouvrage rappellent que le modèle compréhensif, qui s'est lentement développé au sein d'histoires éducatives nationales différentes d'un pays à l'autre, a revêtu des formes diverses avant souvent de se mettre aujourd'hui à décliner, que l'organisation scolaire commune a pu être battue en brèche par la diversification pédagogique et, enfin, que la différenciation curriculaire a pu être entravée par les régulations institutionnelles développées. Ils posent justement, ce faisant, la question de la ségrégation sociale qui a pu résulter de ces politiques éducatives.

Pour ce qui relève de ses apports - originaux - au champ de la recherche en éducation, disons que cet ouvrage - si l'on excepte les comparaisons de textes réglementaires relève selon nous surtout du traitement et de l'interprétation de données disponibles dont PISA- pour développer et fonder scientifiquement des explications qui, fréquemment "limitées " au curriculaire et à l'organisationnel, ont déjà souvent été émises, médiatisées et vulgarisées, "hors recherche" stricto sensu. L'ouvrage ne présente en effet pas vraiment d'enquêtes spécifiques ciblées effectuées par les auteurs susceptibles d'exploitations scientifiques liées à l'objet du livre. D'une façon générale, l'approche comparative qui est, comme son titre l'indique, effectivement bien centrée sur l'école moyenne - tension entre la différenciation mise en place et le commun revendiqué - renvoie aux effets sur l'éducation des contextualisations classiques liées à la classe, au genre, à la "race", etc., mais pas au territoire - encore moins à la territorialité - dont on sait pourtant que, dans la montagne française, et vraisemblablement dans les territoires ruraux isolés, ils « expliquent » environ $15 \%$ de la variance totale de la réussite scolaire contextuelle (Champollion, 2013). Car il ne faut pas oublier que le territorial influe, non seulement sur la densité et la diversité de l'offre de formation, mais aussi et surtout sur le processus pédagogique d'orientation. Il serait à ce propos du reste sans doute nécessaire d'étudier les impacts territoriaux sur l'éducation dans d'autres territoires que montagnards et ruraux. De notre point de vue, enfin, le focus sur l'orientation - point cardinal du débat : la différenciation renforce clairement la gestion sociale par l'orientation - aurait sans doute gagné à être davantage approfondi si l'on se rappelle que l'orientation est encore plus sensible au social que ne le sont les performances scolaires, comme l'ont bien montré successivement notamment Boudon (1973) et Gautier (2011).

Globalement, donc, cet ouvrage se présente principalement à nos yeux comme un bon ouvrage de vulgarisation de la recherche scientifique comparative conduite sur le pan socialement et politiquement controversé de l'école moyenne qui louvoie entre tronc commun et filières. À ce titre, sa contribution nous paraît des plus utiles.

\section{BIBLIOGRAPHIE}

BOUDON R. (1973). L'inégalité des chances : la mobilité sociale dans les sociétés industrielles. Paris : Armand Colin. 
CHAMPOLLION P. (2013). Les inégalités d'éducation et d'orientation d'origine territoriale. Paris :

L'Harmattan.

GAUTIER J. (2011). « En quoi l'école est-elle inégalitaire ? ». Skholé, nº 12.

\section{AUTEURS}

\section{PIERRE CHAMPOLLION}

ECP Lyon-Saint-Étienne, ESO-Caen 